\title{
The Analytic Versus Representational Theory of Measurement: A Philosophy of Science Perspective
}

\author{
(Invited Article) \\ Zoltan Domotor $^{1}$, Vadim Batitsky ${ }^{2}$ \\ ${ }^{1}$ Departments of Philosophy, Biochemistry and Biophysics, University of Pennsylvania, Philadelphia, PA 19104, USA \\ E-mail: zdomotor@sas.upenn.edu \\ ${ }^{2}$ Department of Philosophy, St. John's University, Queens, NY 11439, USA \\ E-mail: batitsky@stjohns.edu
}

In this paper we motivate and develop the analytic theory of measurement, in which autonomously specified algebras of quantities (together with the resources of mathematical analysis) are used as a unified mathematical framework for modeling (a) the time-dependent behavior of natural systems, (b) interactions between natural systems and measuring instruments, (c) error and uncertainty in measurement, and (d) the formal propositional language for describing and reasoning about measurement results. We also discuss how a celebrated theorem in analysis, known as Gelfand representation, guarantees that autonomously specified algebras of quantities can be interpreted as algebras of observables on a suitable state space. Such an interpretation is then used to support (i) a realist conception of quantities as objective characteristics of natural systems, and (ii) a realist conception of measurement results (evaluations of quantities) as determined by and descriptive of the states of a target natural system. As a way of motivating the analytic approach to measurement, we begin with a discussion of some serious philosophical and theoretical problems facing the well-known representational theory of measurement. We then explain why we consider the analytic approach, which avoids all these problems, to be far more attractive on both philosophical and theoretical grounds.

Keywords: Gelfand representation, quantity algebra, state space, observables, measuring process, quantity conversion, quantity restoration.

\section{INTRODUCTION: THEORY-WORLD RELATIONS AND THE MEASUREMENT QUESTION}

O NE OF THE CENTRAL aims of philosophy of science is to formulate a comprehensive and adequate interpretation of how scientific theories relate to the world. Such an interpretation, however, has to face the fact that modern science is dominated by quantitative theories whose mathematical formalism (usually presented in the language of differential or difference equations over real-valued functions) says nothing directly about the world. In order to treat such theories even as potential descriptions of the world, their formalism must be augmented by an extra-mathematical interpretation, in which

(i) all functions appearing in the equations represent amounts, degrees, or intensities of pertinent attributes of natural systems in the theory's intended scope of application, while

(ii) the equations defined over such functions describe the dynamical behaviors of target systems or simply the temporal changes in the amounts or degrees of the systems' pertinent attributes.

Only when interpretations (i) and (ii) are carefully specified, can the theory under consideration be tested by checking whether there is an agreement (to within admissible approximations) between two kinds of quantitative values: (a) those computed for the target system's attributes using the mathematical apparatus of the theory, usually referred to as theoretical predictions, and

(b) those obtained by the experimenters on the basis of measurement interactions with the target system.

All this, of course, is a very familiar (admittedly coarsegrained) outline of theory-world relations. And much of what has been written by philosophers of science takes this outline as a sufficiently precise and informative basis for various topic-specific philosophical explorations of science (e.g., those dealing with scientific realism, scientific explanation and theory testing). What such philosophical explorations seem to ignore, however, is that the above outline of theory-world relations is painfully incomplete because it does not even raise, let alone answer, the following epistemologically and methodologically crucial measurement question:

What is it about natural systems and/or observational interactions therewith that justifies our correlating the system's attributes with such-andsuch numbers?

What makes the foregoing measurement question of considerable foundational significance is the following: Although the world imposes many physical constraints on our observational interactions with natural systems (e.g., we cannot compare the masses of two stars by using an equal-arm balance), it imposes 
no physical constraints whatsoever on how we may correlate various features of the world with numbers. Consequently, unless we can answer the above measurement question, any way of correlating natural systems with numbers could be viewed as a 'measurement result', allowing us to confirm and disconfirm theories at will and, as a result, to turn the very notion of theory testing into nonsense!

While the importance of the measurement question was first recognized by von Helmholtz (1887) and later by Cambell (1920), their answers to this question amounted to little more than informal and general definitions of measurement. In the 1950 s, however, a group of mathematically trained social scientists, building on the earlier work of Hilbert (1899), Hölder (1901) and Stevens (1951), have developed a mathematically precise answer, known as the representational theory of measurement, henceforth acronymed RTM.

\section{RTM AND THE MEASUREMENT QUESTION}

The two central components of RTM are its empiricist philosophical framework and a mathematical method of representation theorems. On the philosophical side, RTM embraces the empiricist methodology and epistemology by aiming to specify and justify the "basic procedures for assigning numbers to objects or events on the basis of qualitative observations of attributes" (Krantz et al. 1971, 2-3, italics ours). In other words, RTM assumes that the natural world, in and of itself, is inherently non-quantitative, and that it is we who 'impose' quantities on the world when we represent various qualitative features of the world by assigning numbers to them. Moreover, the qualitative features of the world which give rise to and justify our numerical assignments are presumed to be observable, where 'observable' means that normal sensory perception would suffice to determine whether some specified qualitative comparison relation holds between two attributebearing objects. ${ }^{1}$

In short, even without stating its philosophical commitments explicitly, RTM seems to be in complete agreement with the empiricist view of measurement as articulated by Rudolf Carnap (the most influential empiricist philosopher of science in the 20th century), who remarked that "Quantitative concepts are not given by nature... It is we who assign numbers to nature... [on the basis of] phenomena [which] themselves exhibit only qualities that we observe." (Carnap 1966, 100, italics in the original).

With these empiricist assumptions in the background, the principal mathematical task of RTM begins with specifying the axioms for (what the theory calls) an empirical or measurement structure, consisting of a domain of attribute-bearing objects of interest together with a qualitative comparison rela- tion thereon and (where applicable) a qualitative composition operation on these objects. For example, in the case of the socalled extensive attributes (such as mass, length, charge, etc.), the empirical structure associated with an attribute $\lambda$ under consideration has the algebraic form $\langle\mathbf{D}, \ominus, \oplus\rangle$, where

(i) $\mathrm{D}$ is a nonempty set representing a collection of physical objects, possessing the extensive attribute $\lambda$ (e.g., if $\lambda$ denotes the length attribute, then $\mathbf{D}$ is a set of lengthbearing rods);

(ii) $\ominus$ is a binary relation on $\mathbf{D}$, modeling a qualitative comparison relation associated with $\lambda$. In particular, for the length attribute the atomic sentence ' $x \ominus y$ ' encodes the observational fact that $\operatorname{rod} x$ is strictly shorter than rod $y$, and its truth value is established operationally. That is to say, sentence ' $x \ominus y$ ' is true provided that when rods $x$ and $y$ are placed side by side with their ends or edges coinciding on one side, on the other side the edge of $\operatorname{rod} x$ is strictly under the edge of $\operatorname{rod} y$; and

(iii) $\oplus$ is a binary operation, encoding a qualitative composition operation on objects in $\mathbf{D}$. For the length attribute $\lambda$, the composite $x \oplus y$ denotes the rod obtained by a collinear juxtaposition of $x$ and $y$.

With the empirical structure $\langle\mathbf{D}, \ominus, \oplus\rangle$ (associated with the attribute $\lambda$ under consideration ) appropriately axiomatized, the next crucial step is to prove that the axiomatically specified order-theoretic and algebraic properties of $\theta$ and $\oplus$, respectively, embody necessary and sufficient conditions for the existence of a real-valued function $f: \mathbf{D} \longrightarrow \mathbb{R}$ (representing the informal notion of assignment of numbers to objects), satisfying the following two conditions for all objects $x$ and $y$ in $\mathbf{D}$ :

1. $x \otimes y \Longleftrightarrow f(x)<f(y)$, and

2. $f(x \oplus y)=f(x)+f(y)$.

In addition, the axioms uphold the uniqueness requirement, asserting that for any function $f^{\prime}: \mathbf{D} \longrightarrow \mathbb{R}$ satisfying (1) and (2) above, there is a real number $c$ such that $f^{\prime}(x)=c \cdot f(x)$.

This kind of mathematical result, available, e.g., in Suppes (1969a), is called a representation theorem for the measurement structure $\langle\mathbf{D}, \ominus, \oplus\rangle$.

From the standpoint of modern algebra, the real-valued structure-preserving function $f$ referred to above is best viewed as a homomorphism or embedding of $\langle\mathbf{D}, \otimes, \oplus\rangle$ into the numerical structure $\langle\mathbb{R},<,+\rangle$. $^{2}$

Having outlined the main ideas and methods of RTM, let us now take a look at how this representational approach proposes

\footnotetext{
${ }^{1}$ For example, in the process of measuring mass on an equal-arm balance, consider placing two objects on its opposite pans and observing whether the balance tilts and, if so, on which side. Here the meaning of 'observable' is that of philosophers and it differs significantly from the way in which this term is used in physics, where it designates a measurable characteristic of a natural system.

${ }^{2}$ Today the literature on RMT includes many excellent surveys (from concise to comprehensive) of the theory's technical aspects. See, for example, Suppes and Zinnes (1963), Krantz (1968), Pfanzagl (1968), Krantz et al. (1971), Narens (1985), Luce and Suppes (2002), to mention just some. Readers interested in detailed proofs of various representation theorems, or in axiomatizations of measurement structures for various attributes may consult any of these texts.
} 
to answer the earlier posed measurement question. ${ }^{3}$ First, representation theorems for various measurement structures can be interpreted as formal assurances that certain qualitative relations/operations involved in our comparison and aggregation of objects in the world are in effect equivalent to certain mathematical relations/operations on numerical values assigned to these objects. Here the point is that measurement-theoretic representation theorems can be seen as mathematically justifying our assignments of quantitative values to amounts of physical attributes by assuring us that (some amount of) the order-theoretic and algebraic structure of real numbers constitutes a 'numerical mirror' of the order-theoretic and algebraic structure of certain specified comparison relations and composition operations on objects having the said attributes. Second, by linking a numerical structure directly to qualitative relations/operations on attribute-bearing objects, RTM justifies the view that their underlying attribute is fundamental in the sense that its measurement requires neither a prior measurement of other attributes nor theoretical assumptions associated with derived measurements.

In brief, the physical-to-mathematical implication established by representation theorems offers a logical constraint on our numerical assignments and thus justifies them as genuine measurement results (in contrast to ad hoc numerical assignments). The mathematical-to-physical implication, on the other hand, explains why we may treat calculated numerical values of physical attributes as being 'answerable' to certain observational interactions with objects, having these attributes. Here 'answerable' is meant in the sense that significant disagreements between calculated (predicted) and measured values of physical attributes are seen as indications that there is something wrong with the theory involving such attributes.

RTM's answer to the measurement question - based on bottom-up empiricist constructions of quantitative representations of physical attributes, is epistemologically very attractive in that it promises to explain and justify the introduction of quantities on the basis of observable qualitative and, thus, epistemically secure features of the world. And a superficial consideration of examples of qualitative relations/operations offered as empirical interpretations of representable measurement structures in the literature on RTM makes the utter transparency of these examples very seductive. After all, what could be epistemologically simpler and more transparent than placing two rods side by side, assuring that their edges coincide on one side and then checking whether the other edge of one rod extends beyond that of the other?

Yet if we take a careful look at the measurement axioms, supporting various representation theorems - e.g., those in the now classic axiomatization of extensive measurement in Suppes (1969a), we will see that the structural properties of relations and operations required for real-valued representations of even such basic attributes as length unavoidably transcend the limits of what is observable. In the next section we will outline several such obstructions to empirical interpretations of measurement axioms. (Our outline will rely on a detailed case study in Batitsky (1998) to which readers are referred for additional details and bibliographic information.)

\section{Problems With RTM's EMPIRICIST FOUNDATION}

Beginning with the comparison relation $\theta$, the transitivity property of this relation requires arbitrarily precise discrimination capacity, which real-world scientific measuring instruments (let alone unaided human sense organs) do not have. Specifically, it is quite easy to set up situations in which human subjects will be asked to compare objects with respect to some extensive attribute and will produce non-transitive perceptual comparison judgments (e.g., when the differences between objects $x$ and $y$, and between objects $y$ and $z$, are below the threshold of sensory detection, while the difference between $x$ and $z$ is sufficiently large to be clearly detectable).

Nor is there any way to give an observational interpretation to the Archimedean property of $\ominus$, which informally tells us that the amount of an attribute possessed by any object can (in principle) be exceeded by aggregating sufficiently many replicas of any other object having this attribute. The problems for empirical interpretability here are quite numerous. First, this property presupposes an unlimited (potentially infinite) supply of 'replicas' for every object. Second, making precise the required formal properties of the relation ' $\mathrm{x}$ is a replica of y' would expose some of these properties (e.g., transitivity) as observationally uninterpretable. Last, but not least, the modal locution "can in principle be exceeded" already goes beyond the empiricist's commitment "to withhold belief in anything that goes beyond the actual, observable phenomena" (van Fraassen 1980, 202, italics ours).

As for the composition operation $\oplus$, its closure property implies a denumerable infinity of attribute-bearing objects in D, which surely goes beyond what can be determined by observation. Moreover, such algebraic properties as positivity involve mereologically meaningless (and thus physically impossible) comparisons of an object $x$ with objects containing $x$ as a part, as in $x \ominus(x \oplus y)$.

Of course, these difficulties with observational interpretation of measurement axioms have not gone unrecognized by measurement theorists who from the beginning clearly saw how much abstraction and idealization is involved in their axiomatizations of measurement structures (e.g., see Suppes 1969a, 44-45). Their response, however, was largely focused on order-theoretic and/or algebraic modifications of the standard axiomatizations, so as to remove observationally uninterpretable properties of $\ominus$ and $\oplus$. For instance, the requirement of (perfect) transitivity for $\ominus$ can be avoided by treating this relation stochastically (Suppes et al. 1989, 300; Kyburg 1997), or by viewing it conservatively as a semiorder relation (in which the atomic sentence ' $x \otimes y$ ' holds only when there is some minimal ('just noticeable') difference between $x$ and $y$ (Domotor and Stelzer 1971). The closure property of $\oplus$ could

\footnotetext{
${ }^{3}$ We emphasize 'proposes' because the adequacy of this answer will be questioned shortly.
} 
be avoided by making this operation partial, i.e., defined only on a proper subset of the domain D (Luce and Marley 1969; Krantz et al. 1971, 81-85). The Archimedean property of Q can be avoided either by switching to finite measurement structures (Suppes 1969, 5-9), whose real-valued representations do not require this property, or to non-Archimedean measurement structures with non-standard real-valued representations (Mundi 1987; Narens 1985, 276ff). Last, but not least, the mereologically meaningless comparisons demanded by the algebraic properties of $\oplus$ could be avoided by imposing certain mereological restrictions on this operation (Batitsky 1998, 59-61).

Upon closer inspection, however, none of these modifications of qualitative relations and operations are amenable to observational interpretation. The general problem with all such modifications of measurement structures is that in removing some observationally uninterpretable properties of the associated relations/operations, these modifications invariably introduce (though not always explicitly) new structural assumptions which are equally resistant to observational interpretation. For example, a stochastic treatment of comparison relations would involve an auxiliary probability structure with an equally idealized comparative relation (e.g., the relation "event $\mathrm{x}$ is strictly less probable than event y", axiomatized as transitive and Archimedean), and other abstract and idealized structural assumptions required for proving the existence of a probability measure (Batitsky 1998, 63). Treating the comparison relation as a semiorder on infinite domains would require enriching this relation with observationally uninterpretable logically higher-order properties, so as to obtain the so-called indiscernibility equivalence relation, mimicking the numerical identity relation. Finite semiordered measurement structures, on the other hand, require highly abstract axioms, e.g., the so-called Scott Condition, which resists even a physical interpretation, let alone an observational one (Batitsky 1998, 62-63).

The cost of making the empirical comparison relation non-Archimedean would be giving up the equivalence of logical structure between physical and mathematical relations/operations. Instead, there would be only a onedirectional implication: either the 'left-to-right' physical-tomathematical implication (Narens 1985, 276) or the 'rightto-left' mathematical-to-physical implication (Mundy 1987). And this means that RTM would no longer offer us either a formal constraint on numerical assignments (by giving up the 'left-to-right' implication) or a formal justification of holding calculated theoretical predictions answerable to measurement interactions with the physical world (by giving up the 'rightto-left' implication). (Batitsky 1998, 60-61; Batitsky 2002, 295-296).

Finally, subjecting the empirical composition operation to mereological constraints (so as to avoid physically meaningless aggregations and comparisons of objects with their proper parts) would require a fair amount of background (Euclidean) geometry needed for defining measurementtheoretically meaningful parts (e.g., parts obtained by dividing a rod in a plane perpendicular to the direction of the rod's length, as opposed to those chipped out of that rod). Such auxiliary geometry, however, requires observationally uninterpretable, highly technical logically second-order structural conditions on the pertinent geometric relations (betweenness, orthogonality, etc.) in order to assure the existence of an Euclidean distance metric (Batitsky 1998, 59-60).

There are other difficulties faced by empirical interpretations of RTM's measurement structures, but those already discussed allow us to conclude that quantities and their measurements cannot be introduced, explained and justified simply by treating them as convenient (and in principle dispensable) proxies for qualitative observable manipulations of physical objects. As we have just seen, such an interpretation would make RTM's axioms for measurement structures literally false and, as a result, deny any extra-mathematical significance to representation theorems supported by such axioms. With this in mind, we are certainly entitled to ask: What exactly then is described by the axioms for these 'measurement' structures? And why should we accept real-valued homomorphisms or embeddings supported by such axioms as explaining/justifying quantitative measurements in science?

One way for RTM to respond (and, as we see it, the only meaningful way) is to abandon its empiricist foundational aims and to present axiomatized measurement structures as theories of quantities, which (as all scientific theories) are entitled to all sorts of idealizations which should be no more objectionable than idealized frictionless surfaces or centers of gravity in physics. If this position is taken, then, however, as we shall discuss next, RTM will have to face several intertwined theoretical disadvantages, which we outline below, so as to motivate our alternative philosophical and theoretical perspectives on measurement in science. (Some of these theoretical disadvantages have been discussed in greater detail by other critics of RTM, e.g., Mari (2000).)

\section{Theoretical Disadvantages of RTM}

\subsection{Theory-dependence of measurement}

Once we see that RTM's measurement structures cannot be given complete qualitative observational interpretations, the distinction between fundamental and derived measurement, central to RTM's empiricist approach, becomes untenable. All measurement in science, even in most pedestrian cases, is ultimately a derived measurement, requiring prior measurements and theoretical background assumptions (laws) which relate different quantities to one another. This theory-dependence becomes especially pronounced when measurement requires a theory-based understanding and specification of (i) interactions between target systems and designated measuring instruments, and (ii) calibration procedures for instruments. ${ }^{4}$ It is

\footnotetext{
${ }^{4}$ Note that even such a simplistic procedure as measuring the length of a rod with a ruler presupposes (among other things) suitable control of the rod's thermal expansion and, thus, an underlying theoretical relationship between length and temperature.
} 
thus of considerable importance for a theory of measurement to be formulated in such a way that its mathematical formalism and the formalism's intended interpretation naturally accommodate the required theoretical relations among quantities. With RTM, however, no such natural accommodation is possible because its formalism offers no mathematically significant constructions relating different measurement structures to one another, or building new models from antecedently specified ones. $^{5}$

\subsection{Error and uncertainty in measurement}

Errors and the associated uncertainty are unavoidable aspects of measurement, and modern science has developed a powerful array of sophisticated engineering and statistical methods for controlling and estimating errors, respectively. Again, it is important for a theory of measurement to be formulated in a way which allows it to account for the role of error control and estimation in scientific measurement. In the framework of RTM, measurement errors have been typically treated in two ways, both of which are theoretically problematic. The first way focuses on weakening the logical structure of relations and operations in a measurement structure, e.g., by replacing weak order with semiorder, so as to account for error-induced intransitive comparisons. This approach is theoretically unsatisfying because it attempts to account for error-induced logical 'imperfections' of measurement results by building these 'imperfections' into what is being measured rather than by making these logical 'imperfections' a feature of our methods and procedures for extracting information about the world through measurement. The second way in which RTM attempts to deal with measurement errors is by 'hiding' them in the representing homomorphism $f: \mathbf{D} \longrightarrow \mathbb{R}$, prompting a probabilistic representation of the form $\mathbf{P}(\alpha \leq f(x) \leq \beta)=p$, which states that the value $f(x)$ of $f$ for object $x$ is between $\alpha$ and $\beta$ with probability $p$. Representational measurement theory has as yet made little progress in isolating the correct probabilistic conditions to be imposed on relational statements, including the product probabilistic transitivity condition, having the form

$$
\operatorname{Pr}(x \ominus y) \cdot \operatorname{Pr}(y \ominus z) \leq \operatorname{Pr}(x \ominus z)
$$

or some other form that jointly with additional conditions grants the existence of a real-valued random variable $f$ together with its probability distribution $\mathbf{P}$ such that the identity

$$
\operatorname{Pr}(x \ominus y)=\mathbf{P}(f(x)<f(y))
$$

holds for all objects $x$ and $y$ in $\mathbf{D}$.

\subsection{The problem of specifying measurands}

Because initial methods and procedures for measuring such basic physical attributes as length, mass and time were developed long before the emergence of theoretical science, one may be tempted to think that, in general, a theory-independent, observation-based understanding of target attributes - the socalled measurands, is always sufficient for developing procedures and methods for measuring these attributes. Such 'bottom-up' approaches to specifying measurands, however, did not fare well even in Newtonian physics, as witnessed by a prolonged confusion between quantities that were eventually distinguished and specified as momentum and kinetic energy. In the social sciences, this approach fares a lot worse. There are many important attributes (e.g., TV violence, intelligence, poverty, fitness, etc.) that come in obvious degrees or perhaps in other less clear comparative manifestations, and thus possess an order structure of some sort, but the representational approach in itself does not provide any clue to their correct measurement. The reason, we believe, is that the actual measurement procedures for these kinds of attributes require considerably deeper (underlying) psychological, sociological, cultural and other theories or models that the representational approach does not engage. For example, if the measurer does not have a viable model for TV violence or human intelligence, it is not clear exactly what his or her TV violence indicators or intelligence tests are measuring. Theoretical models and their discretized variants (discussed later) provide the required meeting ground for measurement design, interpretation, and model validation.

More generally (and closely related to the already discussed theory-dependence of measurement), a measurand tends to be part of a larger network of other measurands to which it is causally linked or statistically correlated, and framed in an appropriate model or theory of the underlying measured object (typically by equations involving partially known parameters). In the absence of well-confirmed theoretically framed measurands, there is a danger of designing spurious measurements of attributes that do not provide any empirical information about them. In sum, decisions about what to measure and how to measure are for the most part theory-dependent or model-driven. In the case of measuring complex attributes, the formal assumptions of RTM require substantive theoretical enrichments that take into account idealizations, and provide an adequate framework for analyzing and interpreting measurement results.

\footnotetext{
${ }^{5}$ We know from Newtonian mechanics that the results of measurement of force applied to a particle are intimately related to the results of measurement of its acceleration (the first derivative of the particle's velocity) and mass. Although conjoint measurement models (with difficult-to-test conditions) attempt to address this problem in the case of simple product constructions, a representational treatment of derivation and other analytic operations on quantities are typically lacking. A particularly simple example which arises frequently is the following: Suppose we are given two extensive measurement structures $\langle\mathbf{D}, \ominus, \oplus\rangle$ and $\left\langle\mathbf{D}^{\prime}, \ominus^{\prime}, \oplus^{\prime}\right\rangle$ for measuring (say) the mass of physical objects in two distinct but overlapping empirical domains $\mathbf{D}$ and $\mathbf{D}^{\prime}$. Let their respective designated representing homomorphisms (embeddings) be $f: \mathbf{D} \longrightarrow \mathbb{R}$ and $f^{\prime}: \mathbf{D}^{\prime} \longrightarrow \mathbb{R}$. Central to measurement is the construction of a joint measurement structure for the union $\mathbf{D} \cup \mathbf{D}^{\prime}$ and that of a corresponding union homomorphism (embedding) $f \cup f^{\prime}$. It is clear that the joint model must involve strong compatibility axioms for relations $\theta$ and $\theta^{\prime}$, but the representational approach does not make them explicit. There are many other important formal constructions that are not available or are unknown in the representational theory, including the existence of various (e.g., tensor, fibered, etc.) products and sums of measurement structures.
} 


\subsection{The problem of finite/discrete versus infinite/continuous}

Because mathematical modeling throughout science relies extensively on the real (and complex) numbers, it is only natural for RTM to focus on real-valued representations. The values of homomorphisms or embedding functions not only are presumed to be sharp, but also can include real numbers (e.g., non-computable or random) which cannot be obtained even in the limit of infinite precision allowed to highly idealized measurement instruments (Batitsky and Domotor 2007, 88-90). With the limited resolution and memory resources of real-world measuring devices, however, numerical values obtained through measurement are (i) at best determined to lie within some relatively small intervals, and (ii) specified only to finitely many decimal places. These considerations strongly suggest that measurement should also be considered in terms of discretizations of continuum models (e.g., as outlined in Batitsky and Domotor (2007, 85-86)), and that the relevant relations between continuum theoretical models and their measurement-induced discretizations should be accounted for by a theory of measurement. Yet again, RTM does not offer any mathematical or conceptual means for accomplishing this task.

Having outlined theoretical disadvantages of RTM, we are now ready to present an alternative approach to measurement. We call this approach 'analytic' because its formal framework is part of mathematical analysis.

\section{THE ANALYTIC THEORY OF MEASUREMENT: CONCEPTUAL BACKGROUND AND INFORMAL INTRODUCTION}

In contrast to RTM, our approach to measurement is based on a realist conception of quantities - the conception according to which

(i) quantities are theoretical entities of science, interpreted as objective characteristics of natural systems, rather than merely our means for convenient representation of an inherently non-quantitative world;

(ii) quantities and their mathematical relations (representing laws) are discovered by scientists, rather than imposed by them on an inherently qualitative world;

(iii) the purpose of measurement is a determination of properties of natural systems, achieved by a physical act of evaluating quantities which produces a real (or complex) number, rather an act of assigning numbers to non-quantitative reality as convenient numerical representations;

(iv) while quantities are instantiated (in various amounts, degrees or intensities) by natural systems, they have 'a life of their own', so to speak. By this we mean that the algebraic, topological and other structures of quantities, as well as functional relations among quantities (e.g., dynamical laws), can be specified and investigated without having to explain what it is about natural systems that makes them instantiate the quantities in the ways they do.

While the foregoing conditions may at first sound metaphysically and epistemologically audacious, this position will emerge a bit later as a natural interpretation of one of the most important results in 20th century mathematics - the socalled Gelfand duality (Gelfand, 1939), one of whose variants we shall use as the mathematical cornerstone of the analytic theory measurement, henceforth acronymed ATM. Before introducing the formal details of ATM, however, we want to give an intuitive and largely informal description of this approach.

Just as painters or fashion designers can think about colors and their combinations independently of physical objects which instantiate them (e.g., colors are described as 'warm' or 'cold', some combinations of colors are described as 'clashing', etc.), we can likewise think about quantities, their 'combinations', and their relations to one another independently of natural systems which instantiate them. We may, for example, consider the ways certain quantities (e.g., mass, velocity) 'combine' to produce certain other quantities (e.g., momentum or kinetic energy). We may consider the ways in which two or more quantities may be 'related' to one another (e.g., as pressure, volume and temperature are related by the ideal gas law).

From a formal point of view, a set of quantities together with some 'natural' ways of combining them to form new quantities constitutes an algebra, which we will call a quantity algebra. In addition to forming an algebra, quantities also possess an essentially unique geometric counterpart - a topological state space (manifold), which not only allows us to represent quantities in terms of continuous (smooth) functions on it, but also provides a correct framework for explaining how quantities work. With this duality between algebraic and topological structures of quantities in mind, we can use quantity algebras for modeling both the time-dependent behavior of a target natural system and its interactions with measuring devices, chosen for measuring certain quantities of interest instantiated by the system. If the measurement is indirect, we use a convenient functional relation between a hard-to-measure measurand and an easy-to-measure quantity to convert the information about the former into information about the latter. The measurand's values are then restored or reconstructed from the instrument's pointer quantity values, using a so-called inverse method. The relation between continuous quantities and discrete outputs of measuring instruments is also described in the same basic algebraic framework, in terms of discretized algebras of quantities and their limits with respected to increasingly refined reading scales.

As for the measurement question posed in the beginning of our paper, it receives a holistic 'top-down' answer from ATM, which reflects the status of all quantities as theoretical entities of science (in contrast to a hard-to-defend foundationalist 'bottom-up' (theory-independent) answer attempted by RTM). Namely, that our measurements on a target system are justified 
by the entire theoretical framework employed in the system's scientific investigations, including (a) theoretical laws which specify functional relations among quantities, (b) theoretical laws which specify the dynamics of system-instrument interactions, and are used for designing and calibrating measurement instruments, (c) mathematical results establishing connections between continuous models and their discretizations (where the latter reflect measurement errors and uncertainties), and more.

The above modeling framework, based on autonomously conceived and algebraically framed quantities, is widely used not only in physics (see, e.g., Bratteli and Robinson, 1987), but also in other disciplines with well-developed methods and strategies for mathematical modeling (e.g., economics, chemistry, mathematical biology, and population dynamics). And although many scientists who use this modeling framework feel that nothing else needs to be added to it mathematically or conceptually, this framework still may be seen as philosophically unsatisfying because it does not explain why quantities associated with a target natural system change the way they do (and, thus, does not really explain why our evaluations of quantities associated with a target natural system receive the values they do). In other words, some philosophers (and philosophically minded scientists) may feel that the quantity algebra framework underlying ATM is instrumentalist in that it can function only as an 'intellectual instrument' for predicting and systematizing the results of measurements. What this framework lacks, it can be claimed, is a philosophically realist explanation of our measurement results in terms of something deeper, pertaining to the modeled natural systems.

As philosophers, we are sympathetic to the realist perspective on science in general and on measurement in particular. And our realist sympathies are completely satisfied by this modeling framework because it does provide us with a realist interpretation of quantity-based models and evaluations of quantities with the help of the earlier mentioned Gelfand duality. Speaking informally (and for now roughly), what Gelfand's celebrated theorem tells us is that every autonomously specified algebra of quantities is representable by (and thus is essentially the same as) an algebra of observables on a uniquely given topological state space (unique up to a homeomorphism), where observables are understood in the scientists' sense, namely as real-valued continuous functions on a state space.

To appreciate the enormous philosophical significance of this result, note that, in modeling natural systems, we certainly can begin (as some scientists do) with a realist commitment to the system's states and state transitions as fundamental entities and processes respectively, and consider the (automatically available) algebra of observables on the system's state space as our algebra of (system-specific) quantities to be used as an algebraic calculational 'instrument' for reasoning about the system's states. On this view, the measured values of observables would be given a realist explanation as being determined by the system's objective internal (not directly accessible) states.

What Gelfand duality shows is that this realist 'underpinning' of measurement need not be assumed outright, but can be completely recovered from models based on autonomously (abstractly) specified algebras of quantities! In other words, if an abstractly specified algebra of quantities is viewed as a formal syntactic framework for scientific modeling, Gelfand duality guarantees a realist geometric semantics for it in terms of state spaces and observables thereon. In doing so, Gelfand duality also makes suspect any philosophical motivations for debating whether states or quantities should be given ontological, epistemological or methodological priority. As we see it, whatever philosophical commitments with respect to states one accepts or rejects (e.g., the commitment to states being real objective entities), such acceptance or rejection will automatically apply (via Gelfand duality) to quantities, and vice versa. And since we better be realists about something that is indicated by measurement results (i.e., we cannot view our measuring instruments as magic boxes spitting out numbers for no reason whatsoever), Gelfand duality makes it perfectly reasonable for us to be realists about both the system's states and its quantities. This is why we said at the beginning of our informal exposition that our realist conception of quantities will emerge as a natural interpretation of Gelfand duality.

Finally, before outlining the formal details of ATM, we should mention that Gelfand duality applies (via its variants) to models of classical systems as well as to models of quantum systems, and with classical systems it also holds for both deterministic and stochastic models. For the sake of simplicity of presentation (and because of space constraints) we will present the formal details of ATM only for models of classical deterministic systems and their classical deterministic measurements, while briefly indicating along the way how these details can be modified to apply in stochastic or quantum modeling.

\section{QUANTITY ALGEBRAs, THEIR DYNAMICS, DISCRETIZATION AND PROPOSITIONAL LOGIC}

In the modern mathematical physics literature (see, e.g., de Groote, 2005, Döring, 2005, and Döring and Isham, 2008), the standard way to reason about the measurement of a classical physical quantity ${ }^{6}$ or more generally about any empirical measurand (e.g., mass, electric charge, potential energy, pressure, stress, viscosity, temperature, humidity, utility, fitness, and so on) is in terms of a real-valued function that can in principle be measured or evaluated by an actual or a physically possible measuring instrument. In technical terms, measurement of a classical target system's characterizing quantity, say

\footnotetext{
${ }^{6}$ Along standard lines, we regard the notion of quantity to be an attribute of a natural system that comes with values (expressed by magnitudes), comprising the attribute's value space.

${ }^{7}$ As alluded to earlier, since not all values of any given 'observable' $\hat{f}$ are presumed to be observable or measurable in any empirical sense, the terminology is at odds with the philosophical idea of observation in science. However, this is the standard term used by most physicists and we retain it.
} 
$f$, is modeled quite simply by a real-valued function of the basic form $\hat{f}: \mathscr{X} \longrightarrow \mathbb{R}$, commonly but misleadingly called the system's observable, ${ }^{7}$ on an underlying measurement domain $\mathscr{X}$ of states, events, objects or some other entities, to the quantity-value or host space $\mathbb{R}$ of reals. To gain a preliminary intuition on which this model is based, recall that in economics the utility quantity $U$ is typically expressed by a real-valued function $\widehat{U}: \mathscr{X} \longrightarrow \mathbb{R}$, where $\mathscr{X}$ denotes a domain of commodities from which a decision maker is invited to choose, and the utility function $\widehat{U}$ encodes (e.g., in some monetary units) the decision maker's utility. Here the question of whether or how $\widehat{U}$ is derived from the decision maker's order-theoretic preference structure does not arise. For another example, the energy quantity $H$ in a simple pendulum in motion is represented by the smooth Hamiltonian observable $\widehat{H}: \mathbb{R}_{/ 2 \pi \mathbb{Z}} \times \mathbb{R} \longrightarrow \mathbb{R}$, where the measurement domain $\mathscr{X}$ is given by the familiar cylinder-shaped topological state space $\mathbb{R}_{/ 2 \pi \mathbb{Z}} \times \mathbb{R}$. In each state $\mathrm{x} \in \mathscr{X}$, the pendulum possesses a unique magnitude of energy, namely $\widehat{H}(\mathrm{x})$, expressed in a dimensionless manner or in some implicitly specified energy units.

Traditionally, a major underlying assumption - prompted by the success of well-established physical theories, is that the magnitudes of quantities ought to be real (or complex) numbers. One major reason for this choice is the long history of abundant use of reals in science. However, from the standpoint of mathematics, a decisive reason is that the host space of reals (or complex numbers) is strongly present in all nontrivial categories of mathematical structures of practical importance in analysis and its applications. Another important reason is that the continuum of reals is blessed with a plethora of empirically vital algebraic and geometric structures.

In order to elucidate the relationship between objects as bearers of quantities further, it is useful to briefly recall Leibniz' duality between them. According to Leibniz' Principle of the identity of indiscernibles and the so-called Principle of extensionality, any given thing is determined by its properties and conversely, any property is (extensionally) specified by the set of all individuals having that property. In this sense we can say that the universe of individual objects and that of their properties are dual to each other. This symmetry idea is of course quite limited since properties reflect only two options: either individual objects possess them or they do not. However, we can profitably extend this thought to quantities that come in various amounts, degrees or other distinguishable manifestations, and can change smoothly, continuously or at least measurably in time (or space). To obtain a workable idea of Leibniz' duality in the world of quantities of systems, it is necessary to algebraicize the notion of quantity and its instantiated values in classical systems in such a way that it upholds their representation in terms of suitable real-valued functions, alluded to earlier.

We retain the notion that a scientific description of physical objects and information about them can be effectively formulated in terms of appropriate collections of quantities that have an algebraic life of their own, and have the special property such that if measured, they produce a magnitude in the form of a real number. Boolean algebras are well-known to formalize the calculus of properties of things in this sense and in full analogy, Banach algebras, $\mathbb{R}$-algebras (i.e., commutative unital linear algebras over $\mathbb{R}$ ), and von Neumann algebras in particular are introduced with a similar purpose in mind in the world of classical (and also noncommutative quantum) physical quantities. Specifically, in place of Boolean operations, Banach and the other closely related topological algebras are equipped with binary operations mimicking addition, multiplication, and multiplication by a scalar. ${ }^{8}$ However, unlike Boolean algebras, quantity algebras tend to be furnished with complex topological-algebra structures and they may even carry operators for differentiation. In addition, they support various (e.g., differential) equational formulations of law-like relations between quantities and are convenient for specifying algebraic dynamical models that represent (continuous, smooth or measurable) temporal changes in the target system's quantities. There may be a concern of having to consider infinitely many basic quantities in an attempt to completely characterize a target system. Surprisingly, as we shall illustrate next, most quantity algebras of practical interest are generated by an astonishingly small list of basic quantities.

A particularly simple concrete example involves the classical thermodynamical theory of (ideal) gases in a vessel. As is well-known, the pertinent basic quantities are pressure $p$, volume $v$ and gas temperature $t$, measurable by three types of measuring instruments of different design and range. These quantities satisfy the ideal gas law $p \bullet v=\kappa \cdot t$, involving a gas constant $\kappa$. In modeling a gas-in-a-vessel system, the algebraic approach recommends to use the real commutative unital $\mathrm{Ba}$ nach algebra $\mathfrak{A}(p, v, t)$ that includes the basic quantities $p, v, t$ together with the trivial unit quantity 1 (that always outputs value 1 when measured), and is in essence equal to the norm closure of the linear space spanned by 1 and all formal product terms $p \bullet v, p \bullet t, v \bullet t, p \bullet v \bullet t, p \bullet p, v \bullet v \bullet v, \ldots$ or more generally all quantity terms of the form $q_{1} \bullet q_{2} \bullet \cdots \bullet q_{n}$, where $q_{i}$ in the product term is $1, p, v$ or $t$. In this way, the quantity algebra $\mathfrak{A}(p, v, t)$ contains not only all linear combinations of the form $\alpha \cdot p+\beta \cdot v+\gamma \cdot t$ involving all basic quan-

\footnotetext{
${ }^{8}$ If two quantities can be measured in the same experiment or on the same target system, we obtain two numbers that can be added, multiplied, and so forth. This immediately leads to the possibility of treating quantities as fundamental primitive entities, instantiated by systems and forming an algebra. Recall that a unital Banach algebra is a complete normed algebra of the form $\langle\mathfrak{A},\|\cdot\|, \mathfrak{o}, \mathbf{1},+, \bullet, \cdot\rangle$ over the reals or complex numbers. Another elegant definition under linear space terminology is as follows: A commutative unital Banach algebra is a Banach space $\langle\mathfrak{A},\|\cdot\|, \mathfrak{o},+, \cdot\rangle$ equipped with a multiplication operation $\bullet$ and a unit $\mathbf{\perp}$ satisfying the norm inequality $\|f \bullet g\| \leq\|f\| \cdot\|g\|$ for all $f, g \in \mathfrak{A}$, and the identity $\|\mathbf{l}\|=1$. A Banach space is a normed linear space $\langle\mathfrak{A},\|\cdot\|, \mathfrak{o},+, \cdot\rangle$ which is also a complete metric space under the distance metric $\varrho(f, g)=\|f-g\|$ for all $f$ and $g$ in $\mathfrak{A}$. The simplest 'concrete' Banach algebras are the finite dimensional matrix algebras and the algebras of real-valued continuous functions on closed intervals with the supremum norm. Additional terminology and results can be found in any textbook on Banach algebras, for instance, in Larsen (1973). Banach algebras offer a near-perfect blend of algebra and analysis.
} 
tities with scalars $\alpha, \beta, \gamma$ in $\mathbb{R}$, but also all linear combinations of arbitrarily complex products of quantities of the form $\alpha \cdot p \bullet v+\beta \cdot v \bullet t+\gamma \cdot t \cdot p$, and so forth. And, finally, the algebra $\mathfrak{A}(p, v, t)$ also includes the limits of all Cauchy sequences of quantities with respect to the norm defined below. For any quantity $f$ in $\mathfrak{A}(p, v, t)$, its norm $\|\cdot\|$ is defined by

$$
\begin{array}{r}
\|f\|={ }_{d f} \inf \left\{\alpha \mid \alpha \cdot \mathbf{1}-f \in \mathfrak{A}_{+}(p, v, t) \&\right. \\
\left.\alpha \cdot \mathbf{1}+f \in \mathfrak{A}_{+}(p, v, t)\right\},
\end{array}
$$

where $\mathfrak{A}_{+}(p, v, t)$ denotes the so-called positive cone of elements of $\mathfrak{A}(p, v, t)$ (i.e., the subset of all quantities $f$ having the square form $f=g^{2}=g \bullet g$ for some $g$ ).

The norm closure condition ensures that the algebra $\mathfrak{A}(p, v, t)$ also includes more complex elements of the form $\varphi(p, v, t)$, where $\varphi: \mathbb{R}^{3} \longrightarrow \mathbb{R}$ is any continuous function. Clearly, if there are measuring instruments for measuring quantities $p, v$ and $t$, then in principle it is possible to construct an instrument that measures the quantity $p+v+t$. All it takes is to enter the results of measurement obtained from the basic quantities into a pocket calculator and add them. Quite similarly, the value of quantity $\varphi(p, v, t)$ is obtained by applying the function $\varphi$ to the measurement outputs for $p, v$ and $t$, using a convenient software for computing the values of $\varphi$.

It should be born in mind that in view of its closure requirements, the quantity algebra $\mathfrak{A}(p, v, t)$ is bound to include many highly complex quantities that are of no practical interest in characterizing the thermodynamical system under consideration. It is of interest to ask what happens, if the algebra comes with algebraic equations relating some of the quantities. In view of the ideal gas law - allowing the (absolute) temperature quantity to be definable in terms of pressure and volume, the algebra must be quotiented out by all terms that are rendered equal. In full analogy with filters of Boolean algebras, each family of equations gives rise to a unique Banach algebra ideal ${ }^{9}$ that in turn specifies a congruence relation on $\mathfrak{A}$, rendering equivalent quantities equal. We will make use of (maximal) ideals in the next subsection when dealing with function algebra representations of quantity algebras.

Upon reverting to the functional representation, we find that in the absence of the gas law, the basic quantities in $\mathfrak{A}(p, v, t)$ are represented by continuous projection functions of the form $\hat{p}, \widehat{v}, \widehat{t}: \mathbb{R}_{+}^{3} \longrightarrow \mathbb{R}$. For example, the pressure observable $\hat{p}$ assigns to each positive triple $(a, b, c)$ of possible values of pressure, volume, and temperature its first coordinate, i.e., $a$. However, in view of the gas law, the measurement domain is given by the strictly smaller subset (hypersurface)

$$
\mathscr{X}={ }_{d f}\left\{(a, b, c) \in \mathbb{R}_{+}^{3} \mid a \cdot b=\kappa \cdot c\right\},
$$

where as above, we have $a=\widehat{p}(a, b, c))$, and similarly for the other quantity values. We postpone the discussion of representation of quantities in terms of state functions until the next Section.

It is important to emphasize that the algebraic-analytic framework for quantities is completely autonomous and selfcontained. Specifically, to each natural system $S$ of interest we assign a minimal quantity algebra $\mathfrak{A}_{\mathrm{S}}$ of an appropriate type, ${ }^{10}$, whose values completely characterize the system from an assumed perspective.

A significant advantage of the algebraic approach that focuses on the topological (smooth or measurable) algebra structure of quantities is its impressive formal flexibility which allows for incorporation of the following three major constructions on Banach as well as other algebras:

\section{Tensor product and compound systems:}

Consider two natural systems $S$ and $S^{\prime}$, described by quantity algebras $\mathfrak{A}_{\mathrm{S}}$ and $\mathfrak{A}_{\mathrm{S}^{\prime}}$, respectively. The description of the compound system $\mathrm{S}+\mathrm{S}^{\prime}$ (consisting of two systems that interact with each other) obeys the fundamental composition law

$$
\mathfrak{A}_{\mathrm{S}+\mathrm{S}^{\prime}} \cong \mathfrak{A}_{\mathrm{S}} \otimes \mathfrak{A}_{\mathrm{S}^{\prime}}
$$

stating that the quantity algebra associated with a compound target system $S+S^{\prime}$ is isomorphic to the tensor product of the respective algebras of constituent systems. In the isomorphism above, a quantity $f$ of system $S$ is identified with the quantity $f \otimes 1$ of the joint system $S+S^{\prime}$, and likewise a quantity $f^{\prime}$ of $S^{\prime}$ is identified with $\mathbf{l} \otimes f^{\prime} .^{11}$ From a physical point of view, measurement of a target system's measurand relies on a measurement coupling in the form of a tensor product of the target system's algebra and the algebra of the measurand's measuring instrument, joint dynamics thereon, and interconnection laws between them. It is important to bear in mind that by necessity or by choice, in general not all quantities in $\mathfrak{A}_{\mathrm{S}}$ are suitable for measurement, and those that are selected for measurement, are usually not measurable directly. In view of a limited set of feasible measurands and their indirect measurements, measurement procedures are bound to provide only partial information about the target system.

Completely similar systems (e.g., two copies of the same system located in different places) possess isomorphic quantity algebras. In general, the assignment of algebras to systems is unique only up to an algebra isomorphism. In this manner, it is possible to study an entire class of stereotype natural systems with the help of an associ-

\footnotetext{
${ }^{9}$ Recall that a linear subspace $\mathfrak{J}$ of a Banach algebra $\mathfrak{A}$ is an ideal in $\mathfrak{A}$ provided that it satisfies the closure condition $f \in \mathfrak{J} \& g \in \mathfrak{A} \Longrightarrow f \cdot g \in \mathfrak{J}$ for all $f$ and $g$ in $\mathfrak{A}$. For later use we also recall that an ideal $\mathfrak{J}$ is said to be maximal just in case $\mathfrak{J} \neq \mathfrak{A}$ and $\mathfrak{J}$ is not contained in any strictly larger ideal.

${ }^{10}$ Commutative unital Banach algebras are usually reserved for continuous quantities of classical physics, the so-called $\mathbb{R}$-algebras are appropriate for smooth quantities, and measurable quantities in the sense of measure theory tend to form a von Neumann or Riesz algebra.

${ }^{11}$ Recall that here 1 denotes the unit of the algebra, encoding the trivial quantity, having a constant value equal to 1 . In this manner the algebras of constituent systems can also be seen as subalgebras of their product algebra.
} 
ated category of quantity algebras and transformations between them.

\section{Time evolution of quantities of isolated dynamical sys-} tems:

In mathematical control and systems theories (see, e.g., Sontag, 1990), the time evolution of an isolated dynamical system is usually characterized by state and measurement equations of the form

$$
\begin{aligned}
\frac{d \mathrm{x}}{d t} & =F(\mathrm{x}(t), \mathrm{u}(t)) \\
\mathrm{m}(t) & =G(\mathrm{x}(t)),
\end{aligned}
$$

where $\mathrm{x}$ is a state variable belonging to an $n$-dimensional smooth manifold $\mathscr{X}, \mathrm{u}$ is a control variable that takes its values in a submanifold of $\mathscr{X}$, the values of measurement function $\mathrm{m}$ belong to an Euclidean space $\mathbb{R}^{m}$, and the functions $F$ and $G$ are both smooth. The first equation is used for computing predictions and the second equation is used for describing measurements.

It has been argued from various sides that what matters is not so much the equations themselves but their solution space, i.e., the algebra (or manifold) of quantities satisfying the equations. Specifically, suppose a quantitative description of the target system $S$ is based on the quantity algebra $\mathfrak{A}_{\mathrm{S}}$. Then the system's deterministic temporal dynamics (semidynamics) is represented by a one-parameter family

$$
\mathfrak{A} \stackrel{\mathfrak{d}_{t}}{\longrightarrow} \mathfrak{A}
$$

of Banach algebra automorphisms (endomorphisms) ${ }^{12}$ of $\mathfrak{A}$ into itself, called dynamical transition maps, where the indexing variable $t$ takes its values in a time group (time monoid) $\mathbb{T}$, which is usually either the additive group of reals $\mathbb{R}$ or that of the integers $\mathbb{Z}$ (the monoid of positive reals or the monoid of natural numbers), satisfying the group-action (monoid-action) laws $\mathfrak{d}_{0}=\mathbf{1}_{\mathfrak{A}}$ and $\mathfrak{d}_{t} \circ \mathfrak{d}_{t^{\prime}}=\mathfrak{d}_{t+t^{\prime}}$ for all $t, t^{\prime} \in \mathbb{T}$.

The foregoing one-parameter family of Banach algebra automorphisms (endomorphisms) together with the underlying Banach algebra $\mathfrak{A}$ is called a dynamical $B a-$ nach algebra (semidynamical Banach algebra $)^{13}$ and is suggestively denoted by $\mathbb{T} \underset{\mathfrak{d}}{ } \mathfrak{A}$. Our main interest is of course in the measurement dynamics on the product algebra $\mathfrak{A}_{\mathrm{S}} \otimes \mathfrak{A}_{\mathrm{M}}$, described by a measurement coupling $\mathfrak{d}_{t}: \mathfrak{A}_{\mathrm{S}} \otimes \mathfrak{A}_{\mathrm{M}} \longrightarrow \mathfrak{A}_{\mathrm{S}} \otimes \mathfrak{A}_{\mathrm{M}}$ between the target system $\mathrm{S}$ and a measuring instrument $\mathrm{M}$. We mention in passing that the so-called crossed product Banach algebras are appropriate for representing the dynamics of time-varying natural systems, characterized by nonautonomous equations.

\section{Discretization of quantity algebras:}

Analytic measurement theory should be able to account for all constituents of a measuring process. In measuring a quantity of interest $f$ on a target system $\mathrm{S}$, described by a quantity algebra $\mathfrak{A}_{\mathrm{S}}$ containing $f$, we need a measuring instrument $M$ that is designed for its measurement. In addition, we need the instrument's associated pointer (output or indicator) quantity ${ }^{14}$ of in $\mathfrak{A}_{\mathrm{M}}$, whose value can be ascertained by reading the instrument. Now, if the measurement interaction has been of the proper kind, then the value of $\circlearrowright f$, read from the instrument $\mathrm{M}$, can be strongly correlated with the unknown numerical value of the measurand $f$, as it existed in the target system $S$ right before the measurement act. It will be useful to allow the value space of $\circlearrowright f$ to be different from that of $f$. For the sake of clarity, it should be mentioned that a measurand $f$ can be measured with several distinct instruments $\mathrm{M}(\circlearrowright f), \mathrm{M}^{\prime}\left(\circlearrowright^{\prime} f\right), \ldots$, designed differently and based on entirely different pointer quantities.

Whatever value the instrument's pointer assumes, due to limited physical discernibility, its reading is inherently coarse-grained. This means, in particular, that the experimenter's reading of the measurement result for $f$ takes place only on the pointer quantity's allocated discrete reading scale $\mathcal{R}_{n}(\circlearrowright f)=\left\{a_{1}, a_{2}, \ldots, a_{n}\right\} \subset \mathbb{R}$ of a finite resolution $n$, specified by the set of rational representative points of a finite (or denumerable) partition (consisting, e.g., of half-open intervals) of the pointer quantity's value space. There are several arguments showing that only discretized versions of quantities are experimentally accessible. ${ }^{15}$

More particularly, each reading scale $\mathcal{R}_{n}(\circlearrowright f)$ induces (by restriction) a discretization (coarse-grained version) $\circlearrowright f_{n}$ of the pointer quantity $\circlearrowright f$ of measurand $f$. What this means is that only the 'readable' values of $\circlearrowright f$ are available, giving rise to the coarse-grained quantity $\circlearrowright f_{n}$. Now, suppose we consider a directed sequence of discretization levels in terms of reading scales $\mathcal{R}_{n}(\circlearrowright f), \mathcal{R}_{n+1}(\circlearrowright f), \ldots$ of increasingly finer degrees of resolution for the pointer quantity $\circlearrowright f$ of $f$ such that

\footnotetext{
${ }^{12}$ Recall that if $\mathfrak{A}$ and $\mathfrak{B}$ are Banach algebras, then a mapping $\mathfrak{d}: \mathfrak{A} \longrightarrow \mathfrak{B}$ between them is a Banach algebra homomorphism provided that it preserves all algebra operations of $\mathfrak{A}$ and obeys the norm inequality $\|\mathfrak{d}(f)\| \leq\|f\|$ for any $f \in \mathfrak{A}$. A Banach algebra endomorphism is a Banach algebra homomorphism from an algebra to itself, and an automorphism is simply a one-to-one and onto endomorphism.

${ }^{13}$ Some authors call it simply a $\mathbb{T}$-Banach algebra or a Banach dynamical system. Algebraic dynamical systems relative to other types of algebras are defined similarly.

${ }^{14}$ In analog devices the pointer quantity of a measurand is typically a (continuous, smooth, etc.) geometric attribute (e.g., length or angle), representing the pointer's possible positions or angles on the dial.

${ }^{15}$ For example, at the information-theoretic level, due to limited memory and other resources, all measurement outcomes must be described by finite means, digitally or by rational number estimates of continuous-scale pointer positions.
} 
at stage $n$ the successor reading scale $\mathcal{R}_{n+1}(\circlearrowright f)$ is obtained from partitions of the elements of its predecessor reading scale $\mathcal{R}_{n}(\circlearrowright f)$. Then, as partitions approach points in the refinement process when the maximum size of the partition intervals goes to zero, the sequence $\circlearrowright f_{n}, \circlearrowright f_{n+1}, \ldots$ of experimentally accessible quantities (gradually increasing the accuracy of measurements) reconstructs $\circlearrowright f$ in the limit. Because the numerical computations of quantity values from equations are also discrete, it is natural to lift the notion of discretization to the world of quantity algebras.

With these brief remarks we recall the following important definition: Given a parent quantity algebra $\mathfrak{A}$ of a target system, a discretization-dediscretization procedure for algebra $\mathfrak{A}$ consists of a directed sequence of finite dimensional quantity algebras (usually comprised of vectors or matrices)

$$
\begin{aligned}
\mathfrak{A}_{\infty} \underset{\mathrm{I}_{n, \infty}}{\stackrel{\Pi_{\infty, n}}{\rightleftarrows}} \mathfrak{A}_{n} & \rightleftarrows \\
& \ldots \rightleftarrows \mathfrak{A}_{2} \underset{\mathrm{I}_{1,2}}{\stackrel{\Pi_{2,1}}{\rightleftarrows}} \mathfrak{A}_{1}
\end{aligned}
$$

where each embedding algebra homomorphism $\mathrm{I}_{n, n+1}$ sends a given approximating finite-dimensional quantity algebra $\mathfrak{A}_{n}$ to the next strictly finer finite-dimensional quantity algebra $\mathfrak{A}_{n+1}$ that in turn is projected by the algebra homomorphism $\Pi_{n+1, n}$ back onto $\mathfrak{A}_{n}$, as the diagram above indicates. This kind of directed sequence of approximating algebras comes with a so-called direct limit quantity algebra $\mathfrak{A}_{\infty}$ that tends to be close enough (in some metric sense) to the parent algebra $\mathfrak{A}$, showing that the parent algebra is adequate and was correctly chosen. The technical notion of a direct limit of the sequence of discretized algebras $\mathfrak{A}_{n}$ that we have in mind here is the one used, e.g., by Benatti and Cappellini (2005), in the category of von Neumann algebras. We conclude this subsection by emphasizing that model validation involves a comparison of measurement results with those predicted by the model. For a valid model, it should always be theoretically possible to recover the model from its directed sequence of discrete offspring models in the limit, as discretization approaches zero.

\section{Geometric logic of quantities:}

Although this is not always made explicit in the literature on classical measurement, in parallel with the assignment of a minimal quantity algebra $\mathfrak{A}_{\mathrm{S}}$ to a target system $\mathrm{S}$, there is also an association of a propositional language $\mathcal{L}_{\mathrm{S}}$, intended for expressing various claims about quantities of $S$ in terms of their values or more generally for making statements about the target system's properties. Propositional languages come in three basic flavors. ${ }^{16}$

In the simplest deterministic and most idealized situation, there is a consideration of an elementary equational evaluation mapping $\bumpeq: \mathfrak{A}_{\mathrm{S}} \times \mathbb{R} \longrightarrow \mathcal{L}_{\mathrm{S}}$ that assigns to each quantity-magnitude pair $(f, a)$ a unique primitive atomic proposition (written in an infix form) $f \bumpeq a$. Its physical meaning under a classical realist interpretation is as follows: Quantity $f$ has a value and that value is equal to $a .^{17}$ In order to be able to reason about the values of quantities in a systematic fashion, axioms are needed in $\mathcal{L}_{\mathrm{S}}$ for deductive inference. For example, it is natural to include among these axioms the additivity law

$$
(f \bumpeq a \& g \bumpeq b) \Longrightarrow f+g \bumpeq a+b
$$

together with an obvious multiplication law, and more generally the composition law

$$
(f \bumpeq a \& g \bumpeq b) \Longrightarrow \varpi(f, g) \bumpeq \varpi(a, b)
$$

for any (continuous, smooth, measurable) function $\varpi: \mathbb{R}^{2} \longrightarrow \mathbb{R}$ and its generalized variants with $n$ arguments. In addition, there is a longer list of logical rules, governing the basic logical connectives. ${ }^{18}$ Details of axioms are not given - they are easy to provide and the interested reader will find some help in various textbooks on the foundations of physics, including, e.g., Beltramenti and Cassinelli (1981). Under a realist interpretation, we do not equate the values of a quantity with its measurement outcomes. We stipulate that measurement outcomes, relying on pointer quantities, provide only a discrete approximation of such values.

A considerably more general and less idealized class of propositions is based on a membership mapping $\notin: \mathfrak{A}_{\mathrm{S}} \times \mathcal{B}_{\mathbb{R}} \longrightarrow \mathcal{L}_{\mathrm{S}}^{\prime}$ that assigns to each quantity $f$ and a Borel measurable subset $\Delta$ of the real line $\mathbb{R}$ the primitive atomic proposition (written suggestively in an infix form) $f \in \Delta$. Its intended physical meaning under the realist interpretation is the following: Quantity $f$ has a value and that value lies in the real Borel subset $\Delta .{ }^{19}$ Reasoning about quantities and their values

\footnotetext{
${ }^{16}$ Remember that quantities are assumed to possess distinguishable degrees or amounts, expressible by numbers, specifying their value spaces.

${ }^{17}$ Here we wish to emphasize that in classical physics quantities are presumed to possess their values independently of whether or not they are measured. In contrast, empiricists interpret propositions of the form $f \bumpeq a$ in a considerably weaker counterfactual manner thusly: If $f$ were measured by a designated measuring instrument $\mathrm{M}$, then the result would be $a$. These two diametrically opposing interpretations are particularly significant in the theory of quantum measurement.

${ }^{18} \mathrm{As}$ adduced in the previous paragraphs, according to the empiricists, if a given quantity $f$ is measured by a measuring instrument $\mathrm{M}$ and $f^{\prime}$ is measured by the instrument $\mathrm{M}^{\prime}$, then conceptually there is a compound measuring instrument $\mathrm{M}+\mathrm{M}^{\prime}$, consisting of $\mathrm{M}, \mathrm{M}^{\prime}$ and a calculator for addition, that measures $f+f^{\prime}$. Somewhat more generally, there is an obvious instrument $\varpi\left(\mathrm{M}, \mathrm{M}^{\prime}\right)$ for any 'well-behaved' function $\varpi: \mathbb{R}^{2} \longrightarrow \mathbb{R}$.

${ }^{19}$ In applications, instead of using arbitrary Borel subsets $\Delta$ it is sufficient to use only closed intervals $[p, q]$ with rational end-points $p \leq q$.
} 
in $\mathcal{L}_{\mathrm{S}}^{\prime}$ requires additional logical axioms, including the conjunction

$$
\left(f \notin \Delta \& f \notin \Delta^{\prime}\right) \Longleftrightarrow f \notin \Delta \cap \Delta^{\prime}
$$

and disjunction

$$
\left(f \notin \Delta \vee f \notin \Delta^{\prime}\right) \Longleftrightarrow f \Subset \Delta \cup \Delta^{\prime}
$$

laws. The associated propositional language $\mathcal{L}_{\mathrm{S}}^{\prime}$ provides a formal framework for constructing propositions about the target system's quantities and its logic delineates the admissible forms of deductive reasoning about them.

In passing, perhaps we should point out that propositions about the target system specified in $\mathcal{L}_{\mathrm{S}}$ (telling us, e.g., that the system's quantity of interest has this or that value) are theoretical. For example, a proposition "energy $\bumpeq 10 \pi$ " about the energy value of a pendulum in motion is perfectly meaningful in classical mechanics, even though it is impossible to verify it by measuring the pendulum's energy quantity. In addition to the finitary nature of pointer readings, there are various instrument errors, including deterministic and probabilistic errors in the instrument's physical components, errors in calibration, and ultimately errors generated by thermodynamical and quantum fluctuations. These errors obstruct the exact individuation of the value $10 \pi$ among continuum many other real numbers in its arbitrarily small nonempty neighborhoods. However, repeated measurements of energy at increasingly finer levels of resolution will output a sequence of nested intervals [30, 40], [31, 32], [31.4,31.5], [31.41, 31.42], . . with rational endpoints that converges to the value $10 \pi$ as the number of consecutively refined measurements of energy goes to infinity. But again, each interval in the sequence provides only a finite amount of information about the real number $10 \pi$ and in view of finitary memory and computational resources, the approximating sequence must be finite, so that convergence belongs to the theory. Thus, as noted earlier, measurement of a quantity is best understood as a reconstruction or approximation procedure that requires a separate propositional language, built over an appropriately discretized quantity-value space. Needless to add, a less useful notion of so-called classical deterministic ideal measurement of a quantity can and is assumed to be absolutely accurate. In this case we have the equivalence

$$
f_{t} \bumpeq a \Leftrightarrow \circlearrowright f_{t+\Delta t} \bumpeq a
$$

stating that if the value of measurand $f$ prior to the measurement act at time instant $t$ is $a$, then the associated pointer quantity's numerical value read from the instrument right after the measurement interaction at $t+\Delta t$ is also $a$, and conversely. As we know, a real-world measurement provides only results of a considerably less informative nature, namely $\circlearrowright f_{n} \bumpeq a$ with a given level of resolution $n$, serving only as an estimate of the actual value of measurand $f$.

Because physical measurements are regularly subjected to uncertainties, the result of measurement is often represented by probabilistic statements of the form $\mathbf{P}(f \in \Delta)=p$, stating that the value of quantity $f$ lies in the real Borel subset $\Delta$ with probability $p$. Technically, the probability measure $\mathbf{P}$ is obtained from a non-negative element of the dual algebra $\mathfrak{A}^{*}$ of $\mathfrak{A}$, by normalization.

The third and epistemically least idealized framework for reasoning about the values of measurands is based on probability density functions defined on a host space. Concretely, to each measurand $f$ in $\mathfrak{A}_{\mathrm{S}}$ we assign a particular probability density function $\mathbf{p}_{f}$ on $\mathbb{R}$ that represents, when $f$ is measured, the information on the possible outcomes of measurement. In the presence of probability density functions, the probability value above is obtained by integration, defined by $\mathbf{P}(f \in \Delta)={ }_{d f} \int_{\Delta} \mathbf{p}_{f}(x) \mathrm{d} x$. The convex space $\mathfrak{D}(\mathbb{R})$ of probability density functions on $\mathbb{R}$ has its own logic - familiar to statisticians, given by mixing, product and conditionalization of probability density functions. The basic notion is simple enough: We start with atomic propositions of the form $f \sim \mathrm{p}$, expressing the fact that the possible values of quantity $f$ are distributed in accordance with the probability density function $\mathrm{p}$. Now, if measurands $f$ and $g$ are statistically independent, meaning the information obtained about the values of $f$ does not affect the information about the values of $g$ and conversely, then the following convolution law holds:

$$
(f \sim \mathrm{p} \& g \sim \mathrm{q}) \Longrightarrow f+g \sim \mathrm{p} * \mathrm{q},
$$

where $\mathrm{p} * \mathrm{q}$ is the convolution of densities $\mathrm{p}$ and $\mathrm{q}$. Of special interest are the familiar rules governing conditional probability density functions, but we do not need to recall them here.

This concludes our brief survey of the algebraic and logical approaches to quantities. Needless to add, there are many more constructions that are important in the algebraic treatment of measurands, but there is no room in this paper to describe them. However, we hope that enough has been said to convince the reader that quantity algebras and associated propositional languages provide a sensible framework for the development of analytical approaches to measurement. We now turn to the state-space semantics of quantities.

\section{State-SPACE SEMANTICS OF QUANTITIES}

It is essential to bear in mind that measurement, to be understood as an objective process of estimating, approximating or reconstructing the numerical values of quantities (intended to capture their manifestations in an object of measurement, phenomenon or some other observed bearer), refers directly 
to (and hence provides information about) the state of affairs of the quantity per se, and only indirectly to its bearer that happens to instantiate it. Clearly, many distinct objects may instantiate a given quantity in identical amounts, degrees or manifestations, so that the corresponding measurement data pertain specifically to the quantity itself and not to its actual or possible bearers. Therefore, what matters in analytic approaches to measurement is not so much the object as a target on which measurement is perfomed, but the object's internal condition or "the way the object is" at a given instant of time.

So, in answering the question "Why does ideal measurement of a given quantity possessed by an object result in a particular value at time $t$ ?", a realist response is that the ideal measurement value is what it is, because the target object happens to be in a particular mode of being or simply in a particular state at time instant $t$ that fully determines the quantity's measurement value in question. Now, if we pursue the matter further and ask "What constitutes a state of an object at $t$ ?", a common reply is that the object's state at $t$ is fully specified by the stock of information, stored in the collection of (ideal) measurement values of all observables, instantiated by the target object at $t$. We see at once that there is an inherent circularity in this form of reasoning about states and quantities manifested in objects.

This apparent circularity is broken by the celebrated Gelfand representation result (see Genfand, 1939), stating in essence that any quantity $f$ in the target system's Banach algebra $\mathfrak{A}$ ( $\mathbb{R}$-algebra) is uniquely representable by a continuous (smooth) real-valued function $\hat{f}: \mathscr{X} \longrightarrow \mathbb{R}$, called the Gelfand transform or the observable of $f$, where $\mathscr{X}$ is a compact Hausdorff topological space (smooth simplectic manifold), uniquely determined by $\mathfrak{A}$, modulo a homeomorphism (diffeomorphism) and interpreted as the target system's state space. ${ }^{20}$ Specifically, the Gelfand representation theorem asserts that any real commutative unital Banach algebra $\mathfrak{A}$ satisfyinhg the $\mathbb{R}$-property is, up to isomorphism, exactly the real unital Banach algebra $\mathbf{C}(\mathscr{X})$ of real-valued continuous functions (with the usual supremum norm) on a compact Hausdorff topological space $\mathscr{X}$, defined (among other options) by the space Max $\mathfrak{A}$ of all maximal ideals of $\mathfrak{A}$ and furnished with the so-called Gelfand topology. The observable $\hat{f}$ associated with quantity $f$ is specified pointwise in terms of characters of maximal ideals: $\hat{f}(\mathrm{x})={ }_{d f} \mathrm{Char}_{\mathrm{x}}(f)$ for all maximal ideals $\mathrm{x}$ in $\mathscr{X}^{21}$

Gelfand representation of quantities in terms of observables suggests that instead of focusing on measurement domains that are comprised of physical objects or individual manifestations of a particular measurand - as common in the representational approach, it is far more effective to specify the measurement domains of quantities universally in terms of state spaces, consisting of states of a target system on which measurements are to be performed, and treat the observables of a target system in a unified manner as real-valued (continuous or smooth) state functions of the form $\hat{f}: \mathscr{X} \longrightarrow \mathbb{R}$.

We will make a systematic use of the Gelfand representation result when dealing with measurements of quantities, and we shall try to maintain a sharp distinction - both in concept and notation, between quantities $f$ in a quantity algebra $\mathfrak{A}$ of a target system and their representation $\hat{f}: \mathscr{X} \longrightarrow \mathbb{R}$ in the corresponding function algebra $\mathbf{C}(\mathscr{X})$, isomorphic to $\mathfrak{A}$. Here a leading mathematical idea is that algebraic structures can be traded for topological ones (playing a semantic role) and conversely. In particular, each additional structure on $\mathscr{X}$ that is defined in terms of its topology can also be defined in terms of the structure of algebra $\mathfrak{A}$, isomorphic to $\mathbf{C}(\mathscr{X})$. And of course conversely: a given topological structure is representable by certain more or less familiar algebraic notions. We mention in passing that a Gelfand-style representation is also applicable in the ambience of $\mathbb{R}$-algebras of smooth quantities and in the framework of von Neumann algebras of measurable quantities. Specifically, any given $\mathbb{R}$-algebra $\mathfrak{A}$ is uniquely represented (up to an $\mathbb{R}$-algebra isomorphism) by the algebra $\mathbf{C}^{\infty}(\mathscr{X})$ of smooth (infinitely differentiable) functions on a smooth manifold $\mathscr{X}$, so that each abstractly given smooth quantity $f$ is uniquely represented by a concrete smooth function $\hat{f}: \mathscr{X} \longrightarrow \mathbb{R}$. (For more details, see González and de Salas, 2003.)

The state space $\mathscr{X}$ (determined uniquely by $\mathfrak{A}$ to within a homeomorphism) is crucial in providing a realist semantics for all basic propositions about quantities in the associated language $\mathcal{L}$. Concretely, a proposition of the form $f \bumpeq a$ (stating that the value of quantity $f$ is $a$ ) is represented by the subset

$$
\llbracket f \bumpeq a \rrbracket=_{d f}\{\mathrm{x} \in \mathscr{X} \mid \hat{f}(\mathrm{x})=a\}
$$

of states in which the associated observable $\hat{f}$ has value $a$. In a similar manner, the proposition $f \in \Delta$ (stating that the value of $f$ lies in the Borel subset $\Delta$ ) is represented by the measurable subset

$$
\llbracket f \oplus \Delta \rrbracket=_{d f}\{\mathrm{x} \in \mathscr{X} \mid \hat{f}(\mathrm{x}) \in \Delta\}=\hat{f}^{-1}(\Delta)
$$

of $\mathscr{X}$. Thus, we have specified a fundamental semantic relation $\llbracket \cdot \rrbracket: \mathcal{L} \longrightarrow$ Sub $\mathscr{X}$ between the propositional calculus of $\mathcal{L}$ and a Boolean algebra of subsets of $\mathscr{X}$. Here the technical details depend on whether $\hat{f}$ is assumed to be continuous,

\footnotetext{
${ }^{20}$ At the risk of being pedantic, perhaps we should point out that the Gelfand representation result is framed within the framework of commutative unital $C^{*}$-algebras over the complex field $\mathbb{C}$. However, under a minor technical constraint $\|f\|^{2} \leq\left\|f^{2}+g^{2}\right\|$ for all quantities $f$ and $g$, known as the $\mathbb{R}$-property, the representation idea works equally well also within the universe of Banach algebras over the field of reals. The interested reader is referred to Nassapoulos (1999) for further details.

${ }^{21}$ Recall that the character Char $_{x}$ of a maximal ideal $\times$ is a unique multiplicative linear functional Char $x: \mathfrak{A} \longrightarrow \mathbb{R}$, defined by Char $\times(f)={ }_{d f} c$ with $f-c \cdot \mathbf{1} \in \mathbf{x}$ for all $f$ in $\mathfrak{A}$, where $\mathbf{1}$ is the unit element of the algebra $\mathfrak{A}$. Under the usual empiricist interpretation, each state $\times$ of the target system is completely specified by pointer readings of all measuring instruments associated with the target system's quantities. But the formal encapsulation of these pointer readings is precisely a Banach algebra ( $\mathbb{R}$-algebra) homomorphism into the real line, given by the character Char ${ }_{x}$ In this way, we can also say with the empiricists that the target system is fully specified by a complete collection of measuring instruments.
} 
smooth or measurable. For example, in the framework of measurable functions, the logic of $\mathcal{L}$ is classical. However, since in the case of continuous observables the subset of states $\llbracket f \bumpeq a \rrbracket$ is a closed subset of the compact topological space $\mathscr{X}$, the resulting logic is intuitionistic (Brouwerian).

We said that in classical measurement all propositions of the form $f \bumpeq a, f \in \Delta, f \sim \mathrm{p}$ and their logical combinations express certain properties of the target system that are either possessed or not by the system, depending on how the system is, independently of any measurement. Thus, properties are objective. This interpretation of system properties compels us to extend the realist interpretation also to states. ${ }^{22}$ The concept of state of a system is essential in determining whether or not a proposition in $\mathcal{L}$ is true. Each state $\mathrm{x} \in \mathscr{X}$ assigns to each atomic proposition ' $f \bumpeq a$ ' a unique truth value. For example, proposition ' $f \bumpeq a$ ' is said to be true about $f$ and its signified property is possessed by the target system that is in state $\mathrm{x}$ exactly when $\hat{f}(\mathrm{x})=a$. Likewise, proposition ' $f \in \Delta^{\prime}$ ' is true about $f$ and its corresponding property is possessed by the system that is in state $\mathrm{x}$ provided that $\hat{f}(\mathrm{x}) \in \Delta$. The truth value of a complex proposition is a logical combination of truth values of its atomic constituents. Finally, a probabilistic measurement result can take the statistical form $\mathbf{P}(\circlearrowright f \in \Delta \mid \mathbf{x})=p$, stating that the value of the pointer quantity of $f$ is in $\Delta$, given that the target system is in state $\mathrm{x}$, with probability $p$.

We turn now briefly to the semantics of probability density functions. Empiricists assume that the state space $\mathscr{X}$ is delivered with its associated convex space $\mathcal{D}(\mathscr{X})$ of probability density functions that are automatically transferred by measurands to their host space $\mathbb{R}$. Probability density functions are interpreted epistemically as states of information about measurands. Since all measurements are taken to be uncertain, the result of measurement is not a deterministic value, but a revised state of information. In a Bayesian spirit, the final state of information about a measurand is a combination of a priori, theoretical and measurement outcome-based states of information.

Having shown how states are determined by quantities, we now recall how (smooth, continuous, measurable) temporal changes of quantities are modeled within the state space framework. A dynamical target system is one which changes in time, and what changes is the state of the system. Following the Newtonian tradition, mathematical systems theorists represent state changes of natural dynamical systems in the language of (ordinary) differential equations that are probed for individual solutions. It was the genius of Henri Poincarè that initiated a systematic study of mutual relations between equations and their solutions, and introduced the so-called qualitative geometric study of solution curves. Of particular importance was the discovery that in a state space setting, solutions of equations can be treated uniformly as time-functions parametrized by initial states. The modern mathematical concept of a deterministic topological (smooth, measurable) dynamical system (dynamical model or flow) formalizes this solution-based approach.

Specifically, given a target dynamical system with its quantity algebra $\mathfrak{A}$ and the associated state space $\mathscr{X}$, and a time group $\langle\mathbb{T}, 0,+\rangle$ (that can be discrete or a continuum), by a (deterministic) topological dynamical model we mean a jointly continuous map of the form $\delta: \mathbb{T} \times \mathscr{X} \longrightarrow \mathscr{X}$ (also known as the model's transition map) such that the following axioms of time-group action hold for all time instants $t, t^{\prime} \in \mathbb{T}$ and for all states $\mathrm{x}$ in $\mathscr{X}$ :

(i) Identity property: $\delta(0, \mathrm{x})=\mathrm{x}$, and

(ii) Group property: $\delta\left(t, \delta\left(t^{\prime}, \mathrm{x}\right)\right)=\delta\left(t+t^{\prime}, \mathrm{x}\right)$.

Because in what follows, time-group actions will be frequently referred to in representing the temporal evolution of target systems' states, henceforth the above-introduced dynamical model $\delta: \mathbb{T} \times \mathscr{X} \longrightarrow \mathscr{X}$ is symbolized more succinctly by the curved time-group action arrow $\mathbb{T} \curvearrowright \mathscr{X}$, common in theoretical physics. An impressively large class of deterministic dynamical models arises from autonomous systems of ordinary first-order differential equations by simple state-parametrizations of their solution spaces. Given an initial state $x$, we immediately obtain the trajectory (orbit or solution curve) $\mathbb{T}(\mathrm{x})=_{d f}\{\delta(t, \mathrm{x}) \mid t \in \mathbb{T}\}$ passing through it. If the time domain $\mathbb{T}$ and the state space $\mathscr{X}$ are both smooth manifolds, and if the transition map $\delta$ is also smooth (i.e., infinitely differentiable), then $\mathbb{T} \underset{\delta}{ } \mathscr{X}$ is called a (deterministic) smooth dynamical model.

In a state space setting, a measurement coupling between a target system $S$ and a measuring instrument $M$, designed for measuring $f$, is described by a transition map $\delta: \mathbb{T} \times \mathscr{X}_{\mathrm{S}} \times \mathscr{X}_{\mathrm{M}} \longrightarrow \mathscr{X}_{\mathrm{S}} \times \mathscr{X}_{\mathrm{M}}$, where $\delta\left(t, \mathrm{x}, \mathrm{x}^{\prime}\right)={ }_{d f}$ $\left(\mathrm{x}, \vartheta_{f}\left(\mathrm{x}, \mathrm{x}^{\prime}\right)\right.$ with an equationally specified entangled instrument state $\vartheta_{f}\left(x, x^{\prime}\right)$ for all states $x$ and $x^{\prime}$. Thus the instrument's pointer quantity has the value $\circlearrowright f_{t+\Delta t}\left(\vartheta_{f}\left(\mathrm{x}, \mathrm{x}^{\prime}\right)\right)$ that is perfectly correlated to that of $f_{t}$, provided that the instrument $\mathrm{M}$ is properly chosen and the design of measurement coupling is theoretically and experimentally correct. Perfect correlation answers the question as to why a given instrument successfully measures its measurand.

It is the power of the Banach algebra perspective on quantities that motivates our next step. Any dynamical model $\mathbb{T} \underset{\delta}{ } \mathscr{X}$ on a given state space $\mathscr{X}$ automatically lifts to a dynamical model on the associated Banach algebra $\mathbf{C}(\mathscr{X})$ of continuous functions by a simple composition of functions. Explicitly, we define $\boldsymbol{\delta}: \mathbb{T} \times \mathbf{C}(\mathscr{X}) \longrightarrow \mathbf{C}(\mathscr{X})$ by setting $[\boldsymbol{\delta}(t, f)](\mathrm{x})=_{d f} f(\delta(t, \mathrm{x}))$ for all $f$ in $\mathbf{C}(\mathscr{X}), t \in \mathbb{T}$ and $\mathrm{x} \in \mathscr{X}$. It is easy to check that $\delta$ satisfies the above-displayed group action axioms. We can now reason about temporal

\footnotetext{
${ }^{22}$ Empiricists define the notion of state independently of quantities as a class of physically equivalent preparation procedures or as a collection of possible continually updated information storages that contain just enough information to enable us to calculate the system's future responses without further reference to the system's history.
} 
changes of quantities in the same way as we do about temporal changes of states. Since $\mathbf{C}(\mathscr{X})$ is a Banach algebra, it is no surprise that we have algebraic dynamical models of the form $\mathbb{T} \underset{\delta}{ } \mathfrak{A}$ for any quantity algebra $\mathfrak{A}$. We mention in passing that the so-called skew-product or cocycle action construction on quantity algebras can be used to represent controlled dynamical systems.

We complete the earlier discussion of state semantics by recalling the notion of state restriction $\mathfrak{A}_{\left.\right|_{\mathscr{D}}}$ of a quantity algebra $\mathfrak{A}$ to the intended subdomain of application $\mathscr{D} \subset \mathscr{X}$. We have neglected to point out that measuring instruments usually do not access all possible values of a measurand. For each measurand, the instantiating target system may be in states that are not accessible by the allocated measuring instrument. Furthermore, the state space $\mathscr{X}$ may include states that are physically never realized. In these situations, it becomes necessary to work with a smaller set of states $\mathscr{D}$, compatible with the instrument's range.

If the quantity algebra $\mathfrak{A}$ is Gelfand-represented by the algebra $\mathbf{C}(\mathscr{X})$ of real-valued continuous functions on a state space $\mathscr{X}$, then its restriction $\mathfrak{A}_{\mid \mathscr{D}}$ to an open subset $\mathscr{D}$ of $\mathscr{X}$ is represented by the restricted algebra $\mathbf{C}(\mathscr{D})$ of real-valued functions in $\mathbf{C}(\mathscr{X})$, defined only on $\mathscr{D}$. Suppose we wish to investigate a quantity $f$ only on a smaller open subset $\mathscr{D}$ of states, prompted by local measurement. Then, using the restricted algebra above, we are free to work with restricted propositions of the form $f_{\left.\right|_{D}} \in \Delta$, represented by the set $\{x \in \mathscr{D} \mid \hat{f}(x) \in \Delta\}$. This approach readily generalizes to other propositional languages, discussed earlier.

An important example of the restriction construct is furnished by the discretization of state spaces. Suppose the state space $\mathscr{X}_{n}$ is a discretization of state space $\mathscr{X}$ at discretization level $n$, obtained from $\mathscr{X}$ by a cellular subdivision, where $\mathscr{X}_{n}$ is defined by the set of centers of its subdivision cells. Then the state restriction algebra $\mathfrak{A}_{\mathscr{X}_{n}}$, Banach isomorphic to $\mathbf{C}\left(\mathscr{X}_{n}\right)$, provides a convenient framework for modeling pointer position readings in a measurement act. We turn now to our final topic of measuring instruments and deterministic measuring processes, both treated from an analytic perspective.

\section{Modeling NATURAL SYSTEMS AND MEASURING INSTRUMENTS WITH QUANTITY ALGEBRAS}

According to the analytic approach, measurement involves three principal constituents: measured object, measuring instrument, and the measuring process.

\subsection{Modeling measurement objects}

In AMT, the measurement object or target system $\mathrm{S}$ is rep- resented by a Banach algebra ( $\mathbb{R}$-algebra) $\mathfrak{A}_{\mathrm{S}}$ of quantities, whose values completely characterize the system. Each quantity $f$ comes with its value space $\operatorname{Val}(f)=_{d f}\{a \mid \exists x[\hat{f}(x)=$ a] $\}$ and state space-interpreted propositions.

The target system's time evolution is described by a dynamical Banach algebra $\mathbb{T} \curvearrowright \mathfrak{A}$, where $\mathbb{T}$ is a suitable time group or time monoid.

In applications, the object of measurement is a physical body, substance, field or any other entity in nature of interest (appropriately isolated from its environment), on which observations and measurements of certain instantiated quantities are to be performed. Under the analytic scheme, measurement of a quantity is intimately tied to the physical interconnection of and interaction between the observed target system and chosen measuring instrument. In previous sections we have presented a dual description of object systems, using the frameworks of quantity algebras and associated state spaces.

\subsection{Models of measuring instruments}

Measurement of a quantity is performed by its earmarked sensor, transducer, gauge or by some other calibrated device. A measuring instrument is typically a man-made physical system, comprised of several smaller and simpler analog, digital or hybrid hardware modules that are often accompanied with software components for quantity-value restoration. As in the case of any empirical system, the instrument's physical subsystems and their interconnections are modeled autonomously by equations in an associated quantity algebra. However, because measuring instruments can be quite complicated, often radical idealizations are necessary in providing a tractable description. Furthermore, because measurement results may be processed by convenient submodules that are treated as legitimate parts of the instrument, there is a complication in determining the correct "cut" for instrument vs. processor separation.

In conformity with modeling natural systems, we assume that the classes of quantities of a target system $S$ and accompanying instrument $M$ are described by respective quantity algebras $\mathfrak{A}_{\mathrm{S}}$ and $\mathfrak{A}_{\mathrm{M}}$. Their measurement interaction is specified in terms of coupling dynamics on a tensor product algebra $\mathfrak{A}_{\mathrm{S}} \otimes \mathfrak{A}_{\mathrm{M}}$, discussed earlier.

Because in applications most quantities of the target system $\mathrm{S}$ are not measurable directly, ${ }^{23}$ there is a need for a joint deterministic (or probabilistic) target system/instrument-based forward model that uniquely specifies for each measurand $f$ an easily measurable and readily interpretable quantity $\circlearrowright f$, instatiated by the assigned instrument $\mathrm{M}$. In simple situations, the quantity $\circlearrowright f$ is explicitly given by the forward model's law-like linear operator $\circlearrowright f=\mathfrak{L}(f)$. However, for complex instruments the conversion operator can be nonlinear and it

\footnotetext{
${ }^{23}$ For example, recall the design scheme of a classical analog voltmeter - intended to measure voltage between two distinct nodes of an electric circuit. Based on electromagnetic and mechanical laws, analog voltmeters are configured in such a way that the unknown measurand (voltage) is linked to a current flow that in turn induces a magnetic flux, which finally generates a mechanical torque that is brought to an equilibrium by the instrument's coil spring. The value of voltage is then determined (or rather approximated) highly indirectly by its pointer position on a calibrated dial - after a torque-spring equilibrium has been reached.
} 
may even include an extra argument for control and other parameters that are required for the model's equational presentation. Quite often such operators are decomposable into factors, representing the instrument's building blocks for basic quantity transformations.

It is assumed that the target system and the measurand's designated measuring instrument are prepared (independently of one another) prior to measurement in their initial states and then coupled together for the measurement act. After a brief time-interval interaction between the target system and the appropriated measuring instrument, when the coupled system has reached an equilibrium, the instrument outputs a number, indicated by the position of its pointer on the instrument's dial or by a digital display that is naively interpreted as the measurand's actual value. Here we have to exercise considerable care in interpreting the measurement result. The point is that since in general the instrument's output provides only a limited amount of information about the measurand, the measurement result can only be viewed as an estimate or approximation of the measurand's value. Needless to add, the numerical value read from the instrument can meaningfully be correlated with the measurand's value only if the measurement interaction has been of the right kind, meaning that the instrument is fully operational, and the design of measurement interaction is theoretically and experimentally correct.

In a state space setting, a typical deterministic forward model, implemented in the design of the measuring instrument under consideration, is specified by a state space map $\ell: \mathscr{X}_{\mathrm{S}} \longrightarrow \mathscr{X}_{\mathrm{M}}$ such that the diagram of functions below commutes:

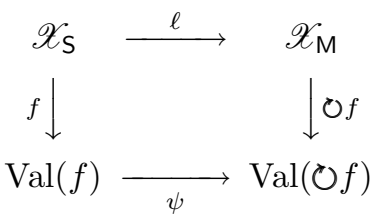

That is to say, the equality $\circlearrowright f \circ \ell=\psi \circ f$ holds, where $\psi$ is a scaling (pointer) function that sends the measurand's values to the values of its associated pointer quantity. As expected, the pointer quantity $\circlearrowright f$ associated with measurand $f$ is taken to be its sole indirect source of measurement data. In particular, the forward model supports the "forward" implication

$$
f \bumpeq a \Longrightarrow \circlearrowright f \bumpeq \psi(a)
$$

stating that if the value of measurand $f$ is $a$, then the value of the instrument quantity $\circlearrowright f$, specified by the forward model, is $\psi(a)$, i.e., a magnitude uniquely determined by $a$. Clearly, in general the converse implication does not hold. For the converse to hold, we need an inverse model's pointer map $\phi: \operatorname{Val}(\circlearrowright f) \longrightarrow \operatorname{Val}(f)$ that justifies

$$
\circlearrowright f \bumpeq b \Longrightarrow f \bumpeq \phi(b) .
$$

However, because the measurement reading is always discrete, having the propositional form $\circlearrowright f_{n} \bumpeq b$ with a given discretization level $n$, it is not sufficient for inferring the exact value of $f$. However, we have a weaker implication of the form

$$
\circlearrowright f_{n} \bumpeq c \Longrightarrow f \notin \Delta_{n}(c),
$$

where $\Delta_{n}(c)$ is the cell or interval of the reading scale $\mathcal{R}_{n}(\circlearrowright f)$ with representative point $c$. Thus, if the instrument reading is $c$, the conclusion is the same statement as $c-\varepsilon \leq \hat{f}(\mathrm{x}) \leq c+\varepsilon$ when then target system is in state $x$.

\subsection{Deterministic measuring process}

As we have already intimated, the classical physical theory of measurement of a quantity is based on the notion of interaction between the measured object and the quantity's instrument that can in principle be made arbitrarily small and any perturbation produced by the interaction (such as energy loss) can be indefinitely weakened or precisely compensated. In this way, we arrive at the concept of ideal measurement that does not disturb the target system's properties. But as we have argued above, even in this ideal setting it is incorrect to take proposition $f \bumpeq c$ to be equivalent to the measurement proposition $\circlearrowright f_{n} \bumpeq c$, where $\circlearrowright f_{n}$ denotes the discretized pointer quantity at discretization level $n$. Simply, the presence of unavoidable errors in meter reading compounds the difficulty of treating measurement propositions as equivalent to the theoretical propositions.

Because measurements are typically indirect or derived, ${ }^{24}$ a deterministic measuring process instantiated in an instrument proceeds in two consecutive stages:

1. Forward conversion stage: Based on well-established physical laws of transformation between suitable system- and instrument-quantities, instantiated by the instrument, in its forward stage the measuring process converts the measurement information about the extant value of the target system's measurand (on which direct measurements may not be possible or feasible within the assumed domain of application) into a more tractable form of information, encoded in a generally simpler and readily interpretable (electro-mechanical, electrochemical, optical, geometric, etc.) instrument quantity. In the case of a classical target system it is assumed that in principle it is possible to measure its measurands without changing the system in any way. Therefore, the outcome realized in the course of measurement and made available via pointer reading is understood to correlate to the pertinent measurand's value immediately preceding the completion of its measurement. (This is of course not the case for quantum systems.)

\footnotetext{
${ }^{24}$ Trivially, even the measurement of weight or mass of a small object is indirect, executed by observing the angular pointer position of a balance scale. Explicitly, the act of measurement relies on a physical law, instantiated by the balance scale, that assigns to mass quantity values appropriate geometric quantity values that are then inverted by calibration and scaling, allowing the observer to receive meaningful information about the measurement outcome in the form of a mass magnitude. Likewise, measurement of mass on an equal-arm balance implicitly assumes the basic laws of mechanics governing equilibrium states. Slightly less trivially, measurement of mass of very large bodies (e.g., the sun) relies on a complex physical theory.
} 
2. Inverse restoration stage: Based on discretization and highly effective digital methods of the so-called inverse problem theory - appropriately realized by the instrument, the post-interaction result of measurement obtained in the forward stage is used in reconstructing, restituting, estimating or approximating the measurand's actual value and providing a convenient access to the measurement outcome.

A simple illustration will help to clarify the restoration stage. We have already indicated that in an ana$\log$ measurement of voltage there is a forward operator that converts voltage via a chain of electro-mechanical quantities into a final geometric displacement of the voltmeter's pointer. By way of the next and last stage of the measurement process, the instrument performs an inversion of the forward operator whose output is no longer continuous. Simply, the act of inversion consists of a discretization of the instrument's continuous quantity, indicated by scale marks that are conveniently dispalyed for meter reading by the experimenter. In sum, the inverse model provides an algorithm for converting a continuous (or smooth) quantity into a discrete quantity. So the most significant thing at this stage is that the measuring instrument provides a direct approximation of the measurand's (unknown) value, expressed by a proposition of the form $f \notin \Delta_{n}(c)$ or its probabilistic variant $\mathbf{P}\left(\circlearrowright f \in \Delta_{n}(c)\right)=p$, discussed earlier.

\section{Concluding Discussion}

The principal aim of this paper is to provide an analytic framework for the study of quantities and their measurement. The motivation comes from a desire to circumvent the theoretical disadvantages of the representational theory of measurement. One way to escape the problems of RTM is to proceed in a 'topdown' manner, via quantity algebras that meet the conditions of the Gelfand representation result. Gelfand representation theorem allows not only to recover the classical state space apparatus of classical systems theory, it ensures a perfect dual equivalence between algebras of quantities and certain topological spaces of states. In this way, abstractly given quantities can be viewed as continuous real-valued functions on a state space. Remarkably, Gelfand representation naturally generalizes also to dynamical quantity algebras in the sense that there is a dual equivalence between algebraic dynamical systems and topological dynamical systems.

Beyond the question of "What are quantities?" that we have answered analytically, lies the question of "How to measure them?". To measure a quantity, often referred to as a measurand, requires a specification of a measuring instrument together with its instrument (pointer or output) quantity, used to read the measurement outcomes. The measuring process is modeled by a physical interaction between the measured object instantiating the measurand and the measurand's measuring instrument. The interaction is described by a state transformation of the compound system "object + instrument," in which the final state of the instrument completely determines the instrument quantity's value that is then used to approximate the measurand's value.

\section{REFERENCES}

[1] Batitsky, V. (1998). Empiricism and the myth of fundamental measurement. Synthese, 116, 51-73.

[2] Batitsky, V. (2002). Some measurement-theoretic concerns about Hale's "Reals by Abstraction". Philosophia Mathematica, 10 (3), 286-303.

[3] Batitsky, V., Domotor, Z. (2007). When good theories make bad predictions. Synthese, 157, 79-103.

[4] Beltramenti, E.G., Cassinelli, G. (1981). The Logic of Quantum Mechanics. Reading, Massachusetts: Addison-Wesley.

[5] Benatti, F., Cappellini, V. (2005). Continuous limit of discrete sawtooth maps and its algebraic framework. Journal of Mathematical Physics, 46, 062702, 1-26.

[6] Bratelli, O., Robinson, D.W. (1987). Operator Albebras and Quantum Statistical Mechanics: Volume 1. New York: Springer.

[7] Campbell, N.R. (1920). Physics: The Elements. Cambridge: Cambridge University Press.

[8] Carnap, R. (1966). Philosophical Foundations of Physics. New York: Basic Books.

[9] de Groote, H.F. (2005). Observables. arXiv:math-ph/0507. 019.

[10] Domotor, Z., Stelzer, J. (1971). Representation of finitely additive semiordered qualitative probability structures. Journal of Mathematical Psychology, 8, 145-158.

[11] Döring, A. (2005). Observables as functions: Antonymous functions. arXiv:math-ph/0510.102.

[12] Döring, A., Isham, C.J. (2008). A topos foundation for theories of physics: I. Formal languages for physics. Journal of Mathematical Physics, 49, 0053515, 1-25.

[13] Gelfand, I.M. (1939). On normed rings. Doklady Akademii Nauk U.S.S.R, 23, 430-432.

[14] González, J.A.N., de Salas J.B.S. (2003). $C^{\infty}$-Differentiable Spaces. Lecture Notes in Mathematics, No. 1824. New York: Springer.

[15] von Helmholtz, H. (1887). Zählen und Messen erkenntnistheoretisch Betrachtet. In Philosophische Aufsätze Eduard Zeller gewidmet. Leibzig: Fuess.

[16] Hilbert, D. (1899). Grundlagen der Geometrie. In Festschrift zur Feier der Enthüllung des Gauss-Weber-Denkmals in Göttingen. Leibzig: Teubner, 1-92.

[17] Hölder, O. (1901). Die Axiome der Quantität und die Lehre vom Mass. Berichte über die Verhandlungen der Königlich Sächsischen Gesellschaft der Wissenschaften zu Leipzig: Mathematisch-Physische Klasse, 53, 1-64.

[18] Krantz, D.H. (1968). A survey of measurement theory. In Danzig, G.B., Veinott, A.F. (eds.) Mathematics of the Decision Sciences: Part 2. Providence, RI: American Mathematical Society, 314-350.

[19] Krantz, D.H., Luce, D., Suppes, P., Tversky, A. (1971). Foundations of Measurement: Volume 1. New York: Academic Press. 
[20] Kyburg, H.E. (1997). Quantities, magnitudes and numbers. Philosophy of Science, 64, 377-411.

[21] Larsen, R. (1973). Banach Algebras. An Introduction. New York: Dekker.

[22] Luce, D., Marley, A.A.J. (1969). Extensive measurement when concatenation is restricted and maximal elements may exist. In Morgenbesser, S., Suppes, P., White, M. (eds.) Philosophy, Science, and Method: Essays in Honor of Ernest Nagel. New York: St. Martin Press, 235-249.

[23] Luce, D., Narens L. (1994). Fifteen problems concerning the representational theory of measurement. In Humphreys, P. (ed.) Patrick Suppes: Scientific Philosopher. Volume 2. Kluwer Academic Publishers, 219-249.

[24] Luce, D., Suppes P. (2002). Representational measurement theory. In Wixted, J., Pashler, H. (eds.) Stevens' Handbook of Experimental Psychology: Volume 4. New York: Wiley, 1-41.

[25] Mari, L. (2000). Beyond the representational viewpoint: A new formalization of measurement. Measurement, 27, 71-84.

[26] Mundy, B. (1987). Faithful representation, physical extensive measurement theory and Archimedean axioms. Synthese, 70, 373-400.

[27] Narens, L. (1985). Abstract Measurement Theory. Cambridge: MIT Press.
[28] Nassopoulos, G.F. (1999). On a comparison of real with complex involutive complete algebras. Journal of Mathematical Sciences, 36, 3755-3765.

[29] Stevens, S.S. (1951). Mathematics, measurement and psychophysics. In Stevens, S.S. (ed.) Handbook of Experimental Psychology. New York: Wiley, 1-49.

[30] Pfanzagl, J. (1968). Theory of Measurement. New York: Wiley.

[31] Sontag, E.D. (1990). Mathematical Control Theory. New York: Springer.

[32] Suppes, P. (1969). Studies in the Methodology and Foundations of Science. Boston: D. Reidel.

[33] Suppes, P. (1969a). A set of independent axioms for extensive quantities. In Suppes (1969) .

[34] Suppes, P., Zinnes, J. (1963). Basic measurement theory. In Luce, R.D. et al. (eds.) Handbook of Mathematical Psychology: Volume 1. New York: Wiley, 3-76.

[35] Suppes, P., Krantz, D.H., Luce, D., Tversky, A. (1989). Foundations of Measurement: Volume 2. New York: Academic Press.

[36] van Fraassen, B. (1980). The Scientific Image. New York: Oxford University Press. 